Pacific Journal of Mathematic 


\title{
EXTENSIONS OF A THEOREM OF LOEWNER ON INTEGRAL OPERATORS*
}

\author{
Donald C. Benson
}

1. Introduction. The purpose of this paper is to extend the result of Loewner in [6]. In that paper the following question is considered A function $k(t)$ which is $L$-integrable on $[0,1]$ is given. He considers the operator

$$
y(t)=-\int_{0}^{1} k(\tau) x(t-\tau) d \tau
$$

applied to continuous functions of period 1. Then $y(t)$ is continuous and also of period 1 , and one may consider the closed oriented curve in the $x y$-plane whose parametric representation is $x=x(t), y=y(t)$. A closed curve is said to have non-negative circulation if the order (index) of the curve with respect to any point not on the curve is non-negative. Now the question is asked: Which kernel functions $k(t)$ generate only curves of non-negative circulation? The answer is given by the following theorem of Loewner: An $L$-integrable function $k(t)$ generates only curves of non-negative circulation if and only if, after a possible change on a set of measure zero, $k(t)$ is analytic in the open interval $0<t<1$ and its derivative can there be represented by a Laplace-Stieltjes integral

$$
k^{\prime}(t)=\int_{-\infty}^{\infty} e^{-s t} d \mu(s)
$$

with a non-decreasing function $\mu(s)$.

The following extensions of this result are of interest:

(i) Consider a function $k(t)$, Lebesgue integrable on the half-line $[0, \infty)$. Apply the operator

$$
y(t)=-\int_{0}^{\infty} k(\tau) x(t-\tau) d \tau
$$

to functions $x(t)$ which are continuous in $(-\infty, \infty)$ and such that

$$
\lim _{t \rightarrow \pm \infty} x(t)=0 \text {. }
$$

Then it is easy to show that $\lim _{t \rightarrow \pm \infty} y(t)=0$, and therefore $x=x(t), y=y(t)$ may be considered as a representation of closed curve. Again we consider the curve $x=x(t), y=y(t)$ and we ask which functions $k(t)$ generate only curves of non-negative circulation.

* Received August 18, 1956, in revised form September 19, 1958. This paper is based upon the author's doctoral dissertation at Stanford University, and was sponsored in part by the United States Air Force under contract No. AF 18 (600) 680. 
(ii) Consider the original problem when all of the integrations are taken in the sense of Cauchy principle value. In particular, we suppose

$$
k(t)=\frac{\phi(t)}{t^{\alpha}(1-t)^{\alpha}}
$$

where $\phi(t)$ is $L$-integrable on the interval $[0,1]$ and $0<\alpha<1$. We apply the integral operator

$$
y(t)=-P \int_{0}^{1} k(\tau) x(t-\tau) d \tau,
$$

to a class of functions $x(t)$ which satisfy a suitable Hölder condition (to insure the existence and continuity of $y(t))$. The symbol " $P$ " denotes that the integration is taken in the sense of Cauchy principal value, i.e.,

$$
P \int_{0}^{1} k(\tau) x(t-\tau) d \tau=\lim _{\substack{\varepsilon \rightarrow 0 \\ \varepsilon>0}} \int_{\varepsilon}^{1-\varepsilon} k(\tau) x(t-\tau) d \tau
$$

Then we may again consider the question: What functions $k(t)$ generate only curves of non-negative circulation?

This problem is particularly interesting for the following reason: Let us suppose that a function is analytic in the interior of the unit circle and continuous on the boundary. Then, on the boundary, the real and imaginary parts of this function are connected by just such an integral relation, where the integral must be taken in the sense of Cauchy principal value. In fact, one has the formula

$$
v(s)=P \int_{0}^{1} u(t) \operatorname{ctg} \pi(s-t) d t
$$

where $u(t)$ and $v(t)$ denote the boundary values of the real and imaginary part respectively, and $t$ denotes the angular displacement from some fixed point on the circle. It is an easy consequence of the argument principle that these curves are of non-negative circulation. It is an indication of what one may expect in general when one considers the formula

$$
-\frac{d}{d t} \operatorname{ctg} \pi s=\csc ^{2} \pi=\frac{1}{\pi} \int_{-\infty}^{\infty} \frac{t e^{-s t}}{1-e^{-t}} d t \text { (see [10], p. 260) . }
$$

Since $\frac{t}{1-e^{-t}}$ is everywhere positive it is clear that the above may be written as a Laplace-Stieltjes integral with non-decreasing determining function.

(iii) We replace the operator $y(t)=-\int_{0}^{1} k(\tau) x(t-\tau) d \tau$ by a general Stieltjes integral 


$$
y(t)=-\int_{0}^{1} x(t-\tau) d k(\tau)
$$

where now $k(\tau)$ is assumed to be a function of bounded variation. This operator is applied to continuous functions $x(t)$ of period 1 . We ask again what functions of bounded variation $k(\tau)$ have the property of always generating curves of non-negative circulation.

The extensions (i), (ii) and (iii) are obtained by methods similar to those of Loewner, and only the results will be stated here. The full details are contained in [1].

In (i) and (ii) Loewner's condition on $k(t)$ is the desired necessary and sufficient condition. In (iii) one must replace (2) by

$$
k^{\prime \prime}(t)=\int_{-\infty}^{\infty} e^{-s t} d \mu(s)
$$

Of course in (i) we must require that the representation (2) is valid in the interval $[0, \infty)$, and, in fact, the integrability of $k(t)$ implies that $\mu(s)$ is constant for negative $s$, so that (2) can in this case be replaced by

$$
k^{\prime}(t)=\int_{0}^{\infty} e^{-s t} d \mu()
$$

(iv) One may consider curves generated by applying the convolution type integral operation to almost periodic functions. It is necessary first to generalize the notion of non-negative circulation to curves given parametrically in terms of almost periodic functions. Then, as before, we ask what kernel functions $k(t), L$-integrable on $[0, \infty)$ have the property that the operator

$$
y(t)=-\int_{0}^{\infty} k(\tau) x(t-\tau) d \tau
$$

always generates curves having this generalized non-negative circulation.

Henceforth we shall be concerned only with (iv).

2. Extension at almost periodic functions. Let us consider curves of the type $x=x(t), y=y(t)$ where the functions $x(t)$ and $y(t)$ are almost periodic functions. Of course, in general, such a curve is not closed. In generalizing Loewner's result, the first problem is to find a suitable generalization of the notion of order or of non-negative circulation. Suppose that $P$ is a point not on the curve. Let $\theta(t)$ denote the argument of the vector from $P$ to the point $(x(t), y(t))$ which is taken so that it is a continuous function of $t$. It is then determined mod $2 \pi$. We may try to define the order of the curve with respect to $P$ as the expression 


$$
c=\lim _{\delta-\gamma \rightarrow \infty} \frac{\theta(\delta)-\theta(\gamma)}{\delta-\gamma}
$$

The trouble is that this limit may not exist. Historically, this constant, when it exists, has been called the mean motion or the secular constant.

In the special case that $P$ is not a limit point of points on the curve, the situation is quite simple. In this case, the limit (9) exists. In fact, Bohr [3] has shown that we may even write

$$
\theta(t)=c t+\phi(t)
$$

where $\phi(t)$ is an almost periodic function.

In the special case that one considers a motion in the complex plane of the form

$$
z(t)=\sum_{\nu=1}^{N} A_{\nu} \exp (i \lambda, t),
$$

with real $\lambda_{\nu}$, the question is completely settled, but by no means simply. In this case the mean motion always exists. This problem was first considered by Lagrange in connection with astronomical questions, who saw the solution to the problem only in the trivial case

$$
\left|A_{1}\right|_{2}^{3}>\sum_{\nu=2}^{N}\left|A_{\nu}\right| .
$$

Later Bohl [4] discovered the existence of a mean motion in the case $N=3$. H. Weyl [7] extended these results to $N=4$ in 1914 (Enseignement Mathématique, vol. 16). In two papers of 1938-39, H. Weyl $[8,9]$ solved the problem for general $N$.

The problem has been considered for analytic functions of a complex variable which are almost periodic in a vertical strip. Many results in this direction are contained in a paper by Jessen and Tornehave [5]. They discuss the mean motions of such functions along a vertical line. They show, among other things, that this type of mean motion does not always exist, even along a vertical line on which the function does not vanish.

In view of the foregoing difficulties I shall avoid the question of existence of mean motions, and shall give an analogue of non-negative circulation which does not use the notion of order with respect to a point.

Definition A. Let $x(t), y(t)$ be defined and continuous for all $t$. Let $\theta_{P}(t)$ denote the argument of the vector from $P$, a point not on the curve $x=x(t), y=y(t)$, to the point $(x(t), y(t))$. We define $\theta_{P}(t)$ so that it is a continuous function of $t$. We say that the curve $x=x(t), y=y(t)$ is of positive sense if there exists a constant $K \leqq 0$ such that for every 
point $P$ not on the curve and for all real numbers $\gamma, \delta(\gamma \leqq \delta)$,

$$
\theta_{P}(\delta)-\theta_{P}(\gamma) \geqq K \text {. }
$$

Definition B. The curve $x=x(t), y=y(t)$ is said to be of weakly positive sense if the above holds for points $P$ not lying on the closure of the curve. The constant $K$ may, in this case, depend on the point $P$.

In the case of continuous periodic functions $x(t), y(t)$ Definitions $\mathrm{A}$ and $\mathrm{B}$ are both equivalent to non-negative circulation. Note that Definition $\mathrm{B}$ is a stronger condition than

$$
\lim _{\delta \rightarrow \gamma \rightarrow \infty} \frac{\theta_{P}(\delta)-\theta_{P}(\gamma)}{\delta-\gamma} \geqq 0
$$

for all points $P$ not belonging to the closure of the curve.

Examples. Let $z=x+i y$. Then the function $z=\exp \left(i|t|^{1 / 2} \sin t\right)$ satisfies (14) but does not satisfy Definitions A or B. The function $z=\left(1+t^{2}\right)^{-1} \exp (i t \sin t)$ satisfies Definition B but does not satisfy Definition A. The function $z=\exp \left(i t+i \sin t^{2}\right)$ satisfies Definition A.

I shall prove the following:

THEOREM 1. Let $k(t)$ be integrable on the interval $[0, \infty)$. Consider the curves generated by applying the operator

$$
y(t)=-\int_{0}^{\infty} k(\tau) x(t-\tau) d \tau
$$

to almost periodic functions. The function $y(t)$ is also almost periodic. $A$ necessary and sufficient condition that the curves generated in this way are of weakly positive sense is that $k(t)$, after a possible change on a set of measure zero, is analytic in $[0, \infty)$, and that $k^{\prime}(t)$ has a representation as a Laplace-Stieltjes integral with non-decreasing determining function, i.e.,

$$
k^{\prime}(t)=\int_{0}^{\infty} e^{-s t} d \mu(s)
$$

where $\mu(s)$ is non-decreasing.

First we prove a lemma similar to Loewner's Lemma 2 [6].

LEMma 1. Let $x_{0}(t), y_{0}(t), x_{1}(t), y_{1}(t)$ be continuously differentiable functions in $(-\infty, \infty)$. Consider the mapping of the strip $0 \leqq s \leqq 1$, $-\infty<t<\infty$ :

$$
\begin{aligned}
& x=(1-s) x_{0}(t)+s x_{1}(t) \\
& y=(1-s) y_{0}(t)+s y_{1}(t) .
\end{aligned}
$$


We suppose that the Jacobian $\frac{\partial(x, y)}{\partial(s, t)}$ is non-negative. Denote the curve $x=x_{0}(t), y=y_{0}(t)$ as $\Gamma_{0}$ and $x=x_{1}(t), y=y_{1}(t)$ by $\Gamma_{1}$. Then if $\Gamma_{0}$ is of positive sense, then so is $\Gamma_{1}$.

Proof. Just as in Loewner's proof, we may assume that $P$ is the image only of points in the st-plane with non-vanishing Jacobian. Indeed, the set of points in the $x y$-plane which are images of points in the st-plane with vanishing Jacobian is of measure zero. In any neighborhood of such a point $P$ exist points $Q$ which are images only of points with positive Jacobian. For such points we shall show the relation (13) for $\Gamma_{1}$ for all $\gamma$ and $\delta, \gamma \leqq \delta$ with fixed $K$. By the continuity of $\theta_{P}$ with respect to the point $P$, (13) will hold also for $P$. A similar consideration shows that it is sufficient to prove that (13) holds when no point on the boundary of the rectangle $R: 0 \leqq s \leqq 1, \gamma \leqq t \leqq \delta$ is mapped into the point $P$. The point $P$ is the image of only finitely many points in $R$, say $P_{1}, P_{2}, \cdots, P_{m}$. We make a triangulation of $R$ satisfying the following conditions:

(i) None of the triangles contains a point $P_{i}$ on its boundary.

(ii) The triangles containing the points $P_{i}$ are mapped into the $x y$-plane in a one-to-one manner, and the Jacobian is there positive.

(iii) The triangles not containing a $P_{i}$ are so small that their images can be included in circles not containing $P$.

Say there are $n$ such triangles. Let us call the images of the boundary curves of triangles, provided with a counter-clockwise orientation, $\sigma_{1}, \sigma_{2}, \cdots, \sigma_{n}$. Let us denote further the order of a curve $\Gamma$ with respect to a point $P$ by $\omega_{P}(\Gamma)$, and let

$$
\underset{s=0}{\Delta} \theta_{P}, \underset{s=1}{\Delta} \theta_{P}, \underset{t=\gamma}{\Delta} \theta_{P}, \underset{t=\delta}{\Delta} \theta_{P}
$$

denote the respective increments of the argument in the counter-clockwise sense of the vector from $P$ to $(x(s, t), y(s, t))$ along each of the four edges of $R$. Then

$$
\sum_{i=1}^{n} \omega_{P}\left(\sigma_{i}\right)=\underset{s=1}{\Delta} \theta_{P}+\underset{s=0}{\Delta} \theta_{P}+\underset{t=\gamma}{\Delta} \theta_{P}+\underset{t=s}{\Delta} \theta_{P} .
$$

Now the images of the segments $t=\gamma, 0 \leqq s \leqq 1$ and $t=\delta, 0 \leqq s \leqq 1$ are straight lines. Hence, we have

$$
\left|\underset{\gamma=t}{\Delta \theta_{P}}\right|<\pi,\left|\underset{t=\delta}{\Delta \theta_{P}}\right|<\pi
$$

The quantities $\omega_{P}\left(\sigma_{i}\right)$ are non-negative. Since $\Gamma_{0}$ is of positive sense we have a constant $K$ (independent of $P$ or $\gamma, \delta$ ) such that $\underset{s=0}{\Delta \theta_{P}} \geqq K$. 


$$
\underset{s=1}{\Delta} \theta_{P} \geqq \underset{s=0}{\Delta} \theta_{P}-\underset{t=1}{\Delta} \theta_{P}-\underset{t=\delta}{\Delta} \theta_{P} \geqq K-2 \pi,
$$

which shows that $\Gamma_{1}$ is of positive sense, since $K$ does not depend on $\gamma, \delta$, or $P$.

In order to show that Theorem 1 makes sense, it is necessary to show that

$$
y(t)=-\int_{0}^{\infty} k(\tau) x(t-\tau) d \tau
$$

represents a continuous functions whenever $x(t)$ is an almost periodic function. I shall show, in fact, that $y(t)$ is an almost periodic function. The continuity of $y(t)$ can be shown as follows. We have for any $t$ and $t^{\prime}$

$$
\begin{gathered}
\left|y(t)-y\left(t^{\prime}\right)\right| \leqq \int_{0}^{\infty}|k(\tau)|\left|x(t-\tau)-x\left(t^{\prime}-\tau\right)\right| d \tau \\
\leqq \sup _{-\infty<\tau<\infty}\left|x(t-\tau)-x\left(t^{\prime}-\tau\right)\right| \int_{0}^{\infty}|k(\tau)| d \tau
\end{gathered}
$$

and since $x(t)$ is uniformly continuous in $(-\infty, \infty)$, we see that $y(t)$ is continuous. To show that $y(t)$ is almost periodic we must further show that there exists a relatively dense set of translation numbers $\tau(\varepsilon)$ for arbitrary $\varepsilon>0$, i.e., there exists a real number $l=l(\varepsilon)$ such that every interval $\alpha<t<\alpha+l$ contains at least one number $\tau(\varepsilon)$ such that for all $t$ we have

$$
|y(t+\tau(\varepsilon))-y(t)| \leqq \varepsilon .
$$

Let $\tau$ be a translation number for $x(t)$ such that

$$
|x(t+\tau)-x(t)| \int_{0}^{\infty}|k(\sigma)| d \sigma<\varepsilon .
$$

Then from (20) we see that this $\tau$ satisfies (21). Further, there exists a relatively dense set of translation numbers $\tau$ satisfying (22). Hence $y(t)$ is almost periodic.

Lemma 2. An n-times differentiable function $f(t)$ such that

$$
f^{(\nu)}(t)(\nu=0, \cdots, n)
$$

are almost periodic can be approximated uniformly up to the nth order by analytic almost periodic functions.

Proof. This follows from the fact that an almost periodic function $f(t)$ whose Fourier series is

$$
f(t) \sim \sum_{\nu=1}^{\infty} A, \exp \left(i \lambda_{\nu} t\right)
$$


can be uniformly approximated by a sequence of functions of the form

$$
S_{q}(t)=\sum_{\nu=1}^{N(q)} c_{q, \nu} A, \exp \left(i \lambda_{\nu} t\right), q=1,2, \cdots .
$$

where the coefficients $c_{q, \nu}$ and $N(q)$ depend on the sequence of Fourier exponents $\lambda_{\nu}(\nu=1,2, \cdots)$, but not on the numbers $A(\nu=1,2,3, \cdots)$, (see Bohr [2], pp. 86-87). If $f(t)$ has almost periodic derivatives, then they have the Fourier series:

$$
f^{(\mu)}(t) \sim \sum_{\nu=1}^{\infty} \lambda_{\nu}^{\mu} A_{\nu} \exp \left(i \lambda_{\nu} t\right)(\mu=1, \cdots, n) .
$$

Thus it appears that the sequences

$$
\begin{aligned}
& S_{q}^{(\mu)}(t)=\sum_{\nu=1}^{N(q)} c_{q, \nu} \lambda_{\nu}^{\mu} A_{\nu} \exp \left(i \lambda_{\nu} t\right) \\
& (\mu=0,1, \cdots, n)(q=1,2, \cdots) .
\end{aligned}
$$

converge uniformly to the derivatives $f^{(\mu)}(t)$. This concludes the proof of Lemma 2.

Now we shall state a lemma which asserts that certain differential operators give rise to curves of positive sense.

Suppose the polynomials

$$
A(r)=a_{0} r^{n}+\cdots+a_{n} \text { and } B(r)=b_{0} r^{n-1}+\cdots+b_{n-1}
$$

satisfy the conditions:

$$
a_{0}>0, b_{0}>0 \text {. }
$$

(28) The roots of $A(r)=0$ and $B(r)=0$ are all real and simple.

(29) The roots of the above equations are mutually separated; i.e.,

if the roots are called $\alpha_{i}(i=1,2, \cdots, n)$ and $\beta_{i}(i=1, \cdots, n-1)$ respectively and if both systems are numbered in ascending order of magnitude, one has

$$
\alpha_{1}<\beta_{1}<\alpha_{2}<\beta_{2}<\cdots<\alpha_{n-1}<\beta_{n-1}<\alpha_{n} .
$$

Now consider a pair of differential operators

$$
\begin{aligned}
& A(f(t))=a_{0} f^{(n)}(t)+\cdots+a_{n} f(t) \\
& B(f(t))=b_{0} f^{(n-1)}(t)+\cdots+b_{n-1} f(t)
\end{aligned}
$$

where the polynomials satisfy the conditions (27), (28), and (29). For $f(t)$ in (31) we put almost periodic functions which possess almost periodic derivatives up to the $n$th order. 


$$
x=A(f(t)), y=B(f(t))
$$

is of positive sense.

The proof of Lemma 3 is similar to Theorem 1 in Loewner [6] and will be omitted.

The next step is to eliminate the function $f(t)$ from the relations (32). Let $x(t)$ be an almost periodic function. There exists an $n$-parameter family of functions $f(t)$ satisfying the differential equation

$$
x(t)=A(f(t)) .
$$

The restriction that $f(t)$ be almost periodic eliminates the indeterminacy. Let $\alpha_{1}, \cdots, \alpha_{n}$ be the roots of the equation $A(r)=0$. We assume $\alpha_{i} \neq 0$. Let $n^{\prime}$ be an integer such that

$$
\begin{aligned}
& \alpha_{i}<0 \text { for } i=1,2, \cdots, n^{\prime} \\
& \alpha_{i}>0 \text { for } i=n^{\prime}+1, \cdots, n .
\end{aligned}
$$

The most general solution of (33) is given as before in the form

$$
\begin{aligned}
f(t)=\sum_{i=1}^{n} c_{i} \exp \left(\alpha_{i} t\right) & +\sum_{i=1}^{n \prime} \eta_{i} \exp \left(\alpha_{i} t\right) \int_{-\infty}^{t} \exp \left(-\alpha_{i} \tau\right) x(\tau) d \tau, \\
& -\sum_{i=n_{1+1}}^{n} \eta_{i} \exp \left(\alpha_{i} t\right) \int_{t}^{\infty} \exp \left(-\alpha_{i} \tau\right) x(\tau) d \tau,
\end{aligned}
$$

where the $c_{i}$ are arbitrary constants and the $\eta_{i}$ are fixed constants depending on the differential equation. Now it is easy to show that the expressions of the form

$$
\exp \left(\alpha_{i} t\right) \int_{-\infty}^{t} \exp \left(-\alpha_{i} \tau\right) x(\tau) d \tau
$$

are almost periodic functions. Similarly the expressions of the form

$$
\exp \left(\alpha_{i} t\right) \int_{t}^{\infty} \exp \left(-\alpha_{i} \tau\right) x(\tau) d \tau, \alpha_{i}>0,
$$

are almost periodic. Since the other terms of (35) are not even bounded, we see that our requirement that $f(t)$ be an almost periodic function implies that the constants $c_{i}$ are all equal to zero. Substitution of this $f(t)$ into the relation $y=B(f(t))$ gives a unique correspondence between $x(t)$ and $y(t)$. The transformation formula is given as follows. We resolve $B(r) / A(r)$ into partial fractions

$$
\frac{B(r)}{A(r)}=\sum_{i=1}^{n} \frac{\omega_{i}}{r-\alpha_{i}} .
$$

From the assumptions (27), (28), and (29) on the polynomials it follows 
that the numbers $\omega_{i}$ are positive, $(i=1, \cdots, n)$. The relation between $x(t)$ and $y(t)$ is of the form

$$
\begin{aligned}
y(t)= & \sum_{i=1}^{n} \omega_{i} \exp \left(\alpha_{i} t\right)\left\{\int_{-\infty}^{t} \exp \left(-\alpha_{i} \tau\right) x(\tau) d \tau+k_{i}\right\} \\
& -\sum_{i=n^{\prime}+1}^{n} \omega_{i} \exp \left(\alpha_{i} t\right)\left\{\int_{t}^{\infty} \exp \left(-\alpha_{i} \tau\right) x(\tau) d \tau+k_{i}\right\}
\end{aligned}
$$

where the constants $k_{i}=0, i=1, \cdots, n$. We will make use only of the special case that the $\alpha_{i}$ are all negative. In this case we have only terms of the form

$$
\omega_{i} \exp \left(\alpha_{i} t\right) \int_{-\infty}^{t} \exp \left(-\alpha_{i} \tau\right) x(\tau) d \tau
$$

and these terms may be expressed in the form

$$
\omega_{i} \int_{0}^{\infty} \exp \left(\alpha_{i} \tau\right) x(t-\tau) d \tau
$$

The kernel function $k^{*}(t)=-\sum_{i=1}^{n} \omega_{i} \exp \left(\alpha_{i} t\right)$ has a derivative with the form

$$
k^{* \prime}(t)=-\sum_{i=1}^{n} \alpha_{i} \omega_{i} \exp \left(\alpha_{i} t\right),
$$

i.e. it is representable as a Laplace-Stieltjes integral with a non-decreasing determining function. In fact, the determining function is here a step function with a finite number of jumps. It is an easily verifiable algebraic fact that given any polynomial $A(r)$ with simple real roots and a positive highest coefficient, a polynomial $B(r)$ can be found such that $A(r)$ and $B(r)$ satisfy the conditions (27), (28), (29) and the $\omega_{i}$ have any preassigned positive values. This means that suitable $A(r)$ and $B(r)$ can be found so that the relation between $x(t)$ and $y(t)$ implied by (32) can be expressed in the form

$$
y(t)=-\int_{0}^{\infty} k(\tau) x(t-\tau) d \tau, k^{\prime}(t)=\int_{0}^{\infty} e^{-s t} d \mu(s)
$$

where $\mu(s)$ is an arbitrarily preassigned non-decreasing step function with finitely many jumps. Such a kernel $k(t)$ will be called a degenerate kernel. These results may be formulated in the following theorem.

Theorem 2. If the kernel $k(t)$ is degenerate, then $k(t)$ generates only curves of positive sense.

(Notice that in Theorem 2 an assertion is made concerning curves of positive sense, whereas Theorem 1 is concerned with curves of weakly positive sense.) 
In order to prove the sufficiency part of Theorem 1 we may carry out a limiting process. Let us assume now that $k(t)$ is an arbitrary $L$ integrable kernel whose derivative has the representation (21). (Of course, since $k(t)$ is integrable on $[0, \infty), \mu(s)$ is continuous at $s=0$.)

In order to show that $k(t)$ generates only curves of non-negative circulation, it is sufficient to show that it can, in the $L_{1}$-mean, be arbitrarily approximated by a degenerate kernel. Indeed, if

$$
\int_{0}^{\infty}\left|k(t)-k^{*}(t)\right| d t<\varepsilon
$$

then the functions

$$
\begin{aligned}
& y(t)=-\int_{0}^{\infty} k(\tau) x(t-\tau) d \tau \\
& y^{*}(t)=-\int_{0}^{\infty} k^{*}(\tau) x(t-\tau) d \tau
\end{aligned}
$$

corresponding to the same continuous function deviate by

$$
\begin{aligned}
\left|y(t)-y^{*}(t)\right| & \leqq \int_{0}^{\infty}\left|k(\tau)-k^{*}(\tau) \| x(t-\tau)\right| d \tau \\
& \leqq M \int_{0}^{\infty}\left|k(\tau)-k^{*}(\tau)\right| d \tau<M \varepsilon
\end{aligned}
$$

where $M$ denotes the maximum of $|x(t)|$. Consider a point $P$ which does not belong to the curve $x=x(t), y=y(t)$ or its closure. The above inequality shows that we can approximate $x=x(t), y=y^{*}(t)$ belonging to a degenerate kernel so closely that

$$
\left|\theta_{P}(x(t), y(t))-\theta_{P}\left(x(t), y^{*}(t)\right)\right|<\mathrm{const} .
$$

Now, since since $x(t), y^{*}(t)$ satisfies Definition $\mathrm{A}$, it follows that $x=x(t)$, $y=y(t)$ is of weakly positive sense. Note that the above argument does not show that $x=x(t), y=y(t)$ satisfies Definition A. It is an open question whether or not in Theorem 1 weakly positive sense can be replaced by positive sense.

The proof that the approximation (40) is possible, is similar to that in Loewner [6, pp. 323-324]. In the present case, certain complication arise because we are considering a infinite interval. The details may be found in [1].

Proof of Theorem 1 (necessity). Suppose that a kernel is $L$-integrable on $[0, \infty)$ and for every almost periodic function $x(t)$, the operator

$$
y(t)=-\int_{0}^{\infty} k(\tau) x(t-\tau) d \tau
$$


generates only curves of weakly positive sense. This implies that for continuous periodic functions of period 1, the operator (44) generates only curves of non-negative circulation. If $x(t)$ is such a periodic function, we have

$$
\begin{aligned}
y(t) & =-\int_{0}^{\infty} k(\tau) x(t-\tau) d \tau=-\sum_{\nu=0}^{\infty} \int_{\nu}^{\nu+1} k(\tau) x(t-\tau) d \tau \\
& =-\int_{0}^{1}\left[\sum_{\nu=0}^{\infty} k(\tau+\nu)\right] x(t-\tau) d \tau
\end{aligned}
$$

where the interchange of summation and integration is justified by the $L$-integrability of $k(t)$. In fact, one has

$$
\int_{0}^{\infty}|k(\tau)| d \tau=\sum_{\nu=0}^{\infty} \int_{\nu}^{\nu+1}|k(\tau)| d \tau=\int_{0}^{1} \sum_{\nu=0}^{\infty}|k(\tau+\nu)| d \tau .
$$

Now, using the theorem of Loewner [6], we have for all $t$ in the interval $0 \leqq t \leqq 1$ except on a set measure zero,

$$
\frac{d}{d t} \sum_{\nu=0}^{\infty} k(t+\nu)=\int_{-\infty}^{\infty} e^{-s t} d \mu(s)
$$

with non-decreasing $\mu(s)$. Replacing $t$ by $t+1$ we have

$$
\frac{d}{d t} \sum_{\nu=1}^{\infty} k(t+\nu)=\int_{-\infty}^{\infty} e^{-s(t+1)} d \mu(s) .
$$

Observing that the difference of two functions differentiable except on a set of measure zero, is differentiable except on a set of measure zero, we have

$$
\frac{d}{d t} k(t)=\int_{-\infty}^{\infty} e^{-s t}\left[1-e^{-s}\right] d \mu(s)=\int_{-\infty}^{\infty} e^{-s t} d \lambda(s)
$$

where $\lambda(s)$ is non-decreasing for $s>0$ and non-increasing for $s>0$. But the integrability of $k(t)$ implies that $\lambda(s)$ must be constant for negative values of $s$. Hence we have the required result

$$
k^{\prime}(t)=\int_{0}^{\infty} e^{-s t} d \lambda(s)
$$

with $\lambda(s)$ non-decreasing. The above formula is now known to be valid only for $t$ in the interval $0 \leqq t \leqq 1$. Application of Loewner's result for arbitrary intervals of the form $(0, n)$ shows that $k^{\prime}(t)$ is analytic on the interval $(0, \infty)$. By analytic continuation, (50) is valid for all nonnegative values of $t$. 


\section{REFERENCES}

1. D. C. Benson, Extensions of a theorem of Loewner on integral operators, Technical Note No. 1, Contract AF 18 (600) 680, Stanford University (1954).

2. H. Bohr, Almost periodic functions, (1947), New York.

3. - Über fastperiodische ebene Bewegungen, Comment. Math. Helv. 4 (1934), 51-64.

4. P. Bohl, Über ein in der Theorie der säkulären Störungen vorkommendes Problem, Crelle's Journal 135 (1909), 189-283.

5. B. Jessen aud H. Tornehave, Mean motions and zeros of almosi periodic functions, Acta Math. 77 (1945), 137-279.

6. C. Loewner, A topological characterization of a class of integral operaiors, Ann. of Math. 49 (1948), 316-332.

7. H. Weyl, Sur une application de la théorie des nombres a la méchanique statistique, Enseignement Mathématique 16 (1914), 455-467.

8. - Mean motion, Amer. J. of Math. 60 (1938), 889-896.

9. Mean motion II, Amer. J. of Math. 61 (1939), 143-148.

10. D. V. Widder, The Laplace transform, (1941), Princeton.

UNIVERSITY OF CALIFORNIA AT DAVIS 



\section{PACIFIC JOURNAL OF MATHEMATICS}

\section{EDITORS}

\section{David Gilbarg}

Stanford University

Stanford, California

\section{R. A. Beaumont}

University of Washington

Seattle 5 , Washington

\author{
A. L. Whiteman
}

University of Southern California Los Angeles 7, California

L. J. Paige

University of California

Los Angeles 24, California

\author{
E. F. BECKENBACH \\ C. E. BURGESS \\ E. HEWITT \\ A. HORN
}

\author{
V. GANAPATHY IYER \\ R. D. JAMES \\ M. S. KNEBELMAN \\ L. NACHBIN
}

ASSOCIATE EDITORS
I. NIVEN

T. G. OSTROM

H. L. ROYDEN

M. M. SCHIFFER
E. G. STRAUS

G. SZEKERES

F. WOLF

K. YOSIDA

\section{SUPPORTING INSTITUTIONS}

\author{
UNIVERSITY OF BRITISH COLUMBIA \\ CALIFORNIA INSTITUTE OF TECHNOLOGY \\ UNIVERSITY OF CALIFORNIA \\ MONTANA STATE UNIVERSITY \\ UNIVERSITY OF NEVADA \\ OREGON STATE COLLEGE \\ UNIVERSITY OF OREGON \\ OSAKA UNIVERSITY \\ UNIVERSITY OF SOUTHERN CALIFORNIA
}

\author{
STANFORD UNIVERSITY \\ UNIVERSITY OF TOKYO \\ UNIVERSITY OF UTAH \\ WASHINGTON STATE COLLEGE \\ UNIVERSITY OF WASHINGTON \\ * * * \\ AMERICAN MATHEMATICAL SOCIETY \\ CALIFORNIA RESEARCH CORPORATION \\ HUGHES AIRCRAFT COMPANY \\ SPACE TECHNOLOGY LABORATORIES
}

Mathematical papers intended for publication in the Pacific Journal of Mathematics should be typewritten (double spaced), and the author should keep a complete copy. Manuscripts may be sent to any one of the four editors. All other communications to the editors should be addressed to the managing editor, L. J. Paige at the University of California, Los Angeles 24, California.

50 reprints per author of each article are furnished free of charge; additional copies may be obtained at cost in multiples of 50 .

The Pacific Journal of Mathematics is published quarterly, in March, June, September, and December. The price per volume (4 numbers) is $\$ 12.00$; single issues, $\$ 3.50$. Back numbers are available. Special price to individual faculty members of supporting institutions and to individual members of the American Mathematical Society: $\$ 4.00$ per volume; single issues, $\$ 1.25$.

Subscriptions, orders for back numbers, and changes of address should be sent to Pacific Journal of Mathematics, 2120 Oxford Street, Berkeley 4, California.

Printed at Kokusai Bunken Insatsusha (International Academic Printing Co., Ltd.), No. 6, 2-chome, Fujimi-cho, Chiyoda-ku, Tokyo, Japan.

PUBLISHED BY PACIFIC JOURNAL OF MATHEMATICS, A NON-PROFIT CORPORATION

The Supporting Institutions listed above contribute to the cost of publication of this Journal, but they are not owners or publishers and have no responsibility for its content or policies. 


\section{Pacific Journal of Mathematics}

\section{Vol. 9, No. $2 \quad$ June, 1959}

Lee William Anderson, On the breadth and co-dimension of a topological lattice

Frank W. Anderson and Robert L. Blair, Characterizations of certain lattices

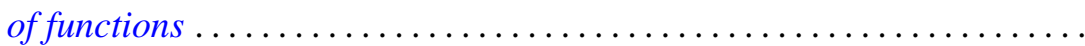

Donald Charles Benson, Extensions of a theorem of Loewner on integral

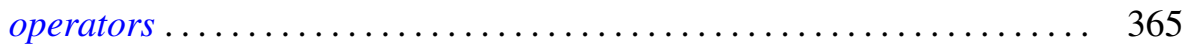

Errett Albert Bishop, A duality theorem for an arbitrary operator ........ 379

Robert McCallum Blumenthal and Ronald Kay Getoor, The asymptotic distribution of the eigenvalues for a class of Markov operators ........

Delmar L. Boyer and Elbert A. Walker, Almost locally pure Abelian

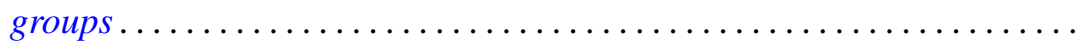

Paul Civin and Bertram Yood, Involutions on Banach algebras ........... Lincoln Kearney Durst, Exceptional real Lehmer sequences .... 415

Eldon Dyer and Allen Lowell Shields, Connectivity of topological

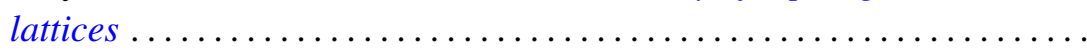

Ronald Kay Getoor, Markov operators and their associated

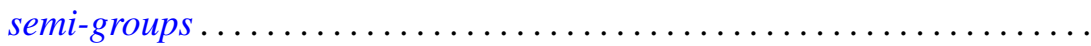

Bernard Greenspan, A bound for the orders of the components of a system of algebraic difference equations

Branko Grünbaum, On some covering and intersection properties in

Minkowski spaces ............................

Bruno Harris, Derivations of Jordan algebras ..............

Henry Berge Helson, Conjugate series in several variables.

Isidore Isaac Hirschman, Jr., A maximal problem in harmonic analysis.

II .

Alfred Horn and Robert Steinberg, Eigenvalues of the unitary part of a matrix

Edith Hirsch Luchins, On strictly semi-simple Banach algebras ...

William D. Munro, Some iterative methods for determining zeros of

functions of a complex variable...

John Rainwater, Spaces whose finest uniformity is metric .

William T. Reid, Variational aspects of generalized convex functions ....

A. Sade, Isomorphisme d'hypergroupoï des isotopes ...... . .

Isadore Manual Singer, The geometric interpretation of a special

connection . . .

Charles Andrew Swanson, Asymptotic perturbation series for characteristic

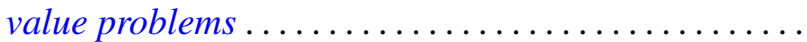

